\title{
UN MODELO DE INTERVENCIÓN DE DESARROLLO COMUNITARIO PARA EL ENVEJECIMIENTO SALUDABLE ${ }^{1}$
}

\section{A COMMUNITY DEVELOPMENT INTERVENTION MODEL FOR HEALTHY AGING}

Fecha recepción: 24 de abril de 2018 / fecha aceptación: 25 de julio de 2019

\author{
Ma de la Luz Martínez Maldonado², Marissa Vivaldo Martínez³, Carolina Angélica \\ González Cuevas ${ }^{4}$ y Juan Pablo Vivaldo Martínez ${ }^{5}$
}

Cómo citar este artículo:

Martínez, M. Vivaldo, Marissa, Gónzalez, Carolina, Vivaldo, Juan. (2019). Un modelo de intervención de desarrollo comunitario para el envejecimiento saludable. Revista Pensamiento y Acción Interdisciplinaria, 5(1), 60-76. DOI: http://doi.org/10.29035/pai.5.1.60

\section{Resumen}

Este estudio parte de la premisa de que más de la mitad de los mayores de 60 años conservan su salud, que no tienen ninguna discapacidad, y que es posible lograr un envejecimiento saludable al enfatizar el capital social con el que cuentan los viejos. En la construcción del modelo de intervención de desarrollo comunitario, empleamos tres herramientas teóricas: la Salud Colectiva: la Sociología de Pierre Bourdieu, y la Promoción de la Salud Emancipadora. El modelo se desarrolló en el marco de la Licenciatura en Desarrollo Comunitario para el Envejecimiento de la Facultad de Estudios Superiores Zaragoza, Tlaxcala, México. Su objetivo principal es mejorar la calidad de vida de personas envejecidas de Tlaxcala, rebasando la perspectiva asistencialista, hacia las personas envejecidas, para reconocerlas como poseedoras de diferentes capitales y como "agentes" capaces de participar y construir sus proyectos. El modelo está integrado por cuatro etapas: diagnóstico, diseño y planificación de la intervención, intervención y evaluación y seguimiento.

\footnotetext{
1 Este proyecto se llevó a cabo gracias al apoyo de PAPIIT IN307516.

2 Facultad de Estudios Superiores Zaragoza, UNAM. Campus 3, Tlaxcala. Psicóloga y maestra en Gerontología Social. Doctora en Ciencias en Salud Colectiva. Miembro del Sistema Nacional de Investigadores (SNI) Nivel 1 y Jefa de la División de Estudios Profesionales Facultad de Estudios Superiores Zaragoza Campus III, Tlaxcala. Miembro de la Unidad de Investigación en Gerontología y responsable de la Línea de Investigación Educación para el Desarrollo Integral Gerontológico. Nacionalidad mexicana. Doctora en Salud Colectiva. E-mail: marilumtz05@gmail.com

3 Facultad de Estudios Superiores Zaragoza, UNAM. Campus 3, Tlaxcala/UNAM. Licenciada en Relaciones Internacionales. Profesor de Asignatura A en la Licenciatura en Desarrollo Comunitario para el Envejecimiento. Miembro de la Unidad de Investigación en Gerontología. Nacionalidad, mexicana. Doctorante en Salud Colectiva. E-mail: marissa.vivaldo@gmail.com 4 Facultad de Estudios Superiores Zaragoza, UNAM. Campus 3, Tlaxcala/UNAM. Licenciada en Historia, Maestra en Ciencias Sociales y Doctorante en Ciencias Sociales. Profesor de Asignatura A en la Licenciatura en Desarrollo Comunitario para el Envejecimiento. Nacionalidad mexicana. E-mail: carolina.cotz@gmail.com

5 Facultad de Estudios Superiores Zaragoza, UNAM. Campus 3, Tlaxcala/Centro de Enseñanza para Extranjeros, UNAM. Licenciado, maestro y doctor en Historia. Profesor de asignatura nivel A en la Facultad de Estudios Superiores Zaragoza y nivel B en el Centro de Enseñanza para Extranjeros. Nacionalidad mexicana. E-mail: jpvivaldo@gmail.com
} 
Los resultados muestran que las prioridades de las personas se centran en el fortalecimiento de sus capacidades para integrarse a la sociedad a través del uso de las tecnologías, en reforzar su autoestima, así como a través del fortalecimiento de estrategias para empoderarse. La intervención comunitaria anclada en programas de investigación ofrece la oportunidad de basar la propuesta de políticas públicas en un cuerpo sistematizado de datos de investigación.

Se concluye que reconocer a la cultura, la historia y la participación de la comunidad como elementos esenciales para la planificación, ofrece la oportunidad de que las personas envejecidas se impliquen en los procesos de capacitación e investigación y participen activamente en el mejoramiento de su salud. El modelo se basa en una implementación sistematizada de cada una de sus diferentes etapas, estando sujeto a una constante evaluación, lo que posibilita implementar adecuaciones inmediatas durante el proceso.

Palabras claves: Adultos mayores, Bourdieu, Salud colectiva, Promoción de salud emancipadora.

\begin{abstract}
This paper is based on the tenet that more than half of the population above 60 years is in good health, does not have major disabilities and that it is possible for them to achieve healthy ageing, as long as their social capital is acknowledged and enforced. As part of the construction of a gerontological community development model, we have turned to three theoretical paradigms: the Collective Health paradigm: Pierre Bourdieu's Sociology, and that of Emancipatory Health Promotion. The model was developed in the framework of the Undergraduate Program on Community Development for Ageing of the School of Higher Studies Zaragoza of the National Autonomous University of Mexico, at Tlaxcala, Mexico. Our main objective is to improve the life quality of elder populations in Tlaxcala beyond current assistance-based approaches, understanding them as individuals endowed with multiple forms of capitals as well as "agents" capable of participating in the construction of their individual life projects. The model is structured according to four stages: diagnostic, intervention design and planning, evaluation, and follow-up.
\end{abstract}

Results demonstrate that the priorities of this population focus on a large extent on the improvement of their ability to integrate to their society through their familiarization with technological relevant knowledge, the reinforcement of their self-esteem, and the development of empowerment strategies. Furthermore, community intervention in parallel with action research offers the possibility of anchoring public policies on a sound corpus of empirical data.

It is concluded that the recognition of the culture, history and forms of participation of the community as key elements in the planning of intervention programs, is associated to the involvement of the elderly in both the training and the research processes, thus enhancing their active commitment in the promotion of a healthy status. The model is based on the systematic implementation of each one of its different components and is subject to a permanent evaluation, which enables the implementation of relevant and contingent changes throughout the process.

Keywords: Elderly; Bourdieu; Collective Health; Emancipatory Health Promotion. 


\section{Antecedentes Generales}

En México, y debido a la transición epidemiológica y demográfica, el número de personas mayores de 60 años se ha incrementado cada vez más, de tal suerte que se pronostica que para las próximas dos décadas aumenten en más del 15\%. Sin duda, esta situación complicará el panorama sanitario, económico y social, por lo que resulta imperativo generar una serie de conocimientos científicos, la formación de recursos humanos y de modelos de intervención para hacer frente a este nuevo reto.

En México, el 10.5\% de la población (alrededor de 12.9 millones de personas) tiene más de 60 años de edad, y se proyecta que para el 2050, el 27.7\% (33.7 millones de personas) pertenecerán a este grupo etario. La esperanza de vida al nacer es de 75.47 años (73 para los hombres y 78 para las mujeres). La esperanza de vida a los 60 años es de alrededor de 22 años ( 21 años para hombres y 24 años para mujeres) (INEGI, 2015).

Estudios recientes reportan que más del 50\% de los mayores de 60 años presenta hipertensión arterial, y un tercio de ellos desconoce el diagnóstico. También se ha encontrado que más del $20 \%$ de los mayores de 60 años tiene diagnóstico de diabetes mellitus tipo 2, de los cuales un alto porcentaje está descontrolado, aunque asista periódicamente al médico; así como, que la mayoría de consultas y servicios de hospitalización que brinda el sector salud, es para el grupo de mayores de 60 años (Gutiérrez, 2012).

Respecto de las condiciones de las personas que envejecen, se ha señalado que más de la mitad de los adultos mayores de 60 años conserva la salud y no tiene discapacidad alguna, lo que representa un valioso capital social, así como una gran oportunidad para fortalecer las acciones de prevención (Gutiérrez\&Lezana, 2013).

Datos recabados por el Instituto Nacional de Estadística y Geografía (INEGI) muestran que de la totalidad de personas mayores de 60 años, $72.6 \%$ cuenta con acceso a la atención de la salud; $77.4 \%$ sabe leer y escribir y está en posibilidades de redactar un mensaje breve; para el $85 \%$ de ellos, no representa dificultad realizar actividades de la vida diaria (básicas, instrumentales o avanzadas); mientras que $79.2 \%$ de las mujeres y $81.5 \%$ de los varones perciben que su salud se ubica en el rango de "regular a buena". Esto constituye, sin lugar a duda, un capital social valioso para generar modelos de intervención.

Ya en otro texto, hemos subrayado la importancia de que diferentes organismos internacionales propongan nuevos enfoques, herramientas y estrategias para enfrentar las demandas que representan los cambios demográficos. Es el caso de la Organización Mundial de la Salud (OMS) que se ha planteado desarrollar un nuevo paradigma para estudiar y analizar el envejecimiento y la vejez, sin que 
se vinculen con pérdidas o deterioros derivados de la jubilación o la enfermedad (Martinez\&Chapela\&Mendoza, 2009). Sin embargo, tales prejuicios no reflejan la realidad actual, pues de acuerdo con las estadísticas, más del 70\% de la población mayor de 60 años es funcional y totalmente independiente (INEGI, 2015), por lo que puede participar en programas a nivel comunitario. Las personas adultas mayores constituyen un capital social determinante para prolongar la esperanza de vida saludable en la vejez a través del autocuidado, la ayuda mutua y la autogestión. Lo que se busca alcanzar, es un envejecimiento saludable, por esta razón, se ha diseñado un modelo de intervención de desarrollo comunitario para las personas en proceso de envejecimiento.

\section{Aproximación Teórica - Referencial}

Para comprender los procesos de participación de los viejos y las acciones que las instituciones destinan a ellos, empleamos dos teorías principales: la Salud Colectiva, la Sociología de Pierre Bourdieu y la Promoción de la Salud Emancipadora. Estos marcos de análisis ofrecen una serie de conceptos, postulados y metodologías, que en conjunto, permiten comprender el envejecimiento y la vejez desde una perspectiva interdisciplinaria.

La Salud Colectiva se ha desarrollado como un campo de conocimiento incluyente que busca analizar desde perspectivas críticas aquellos temas, sujetos y objetos que consistentemente han sido invisibilizados por los paradigmas hegemónicos. Su planteamiento básico es que "las condiciones materiales de existencia determinan la distribución desigual de la salud-enfermedad en los grupos humanos" (Mendoza \& Jarillo, 2011, p. 850). Los objetos de estudio de la Salud Colectiva en relación con la salud son: las necesidades de los grupos sociales, la organización de los servicios y su gestión. Adicionalmente, la Salud Colectiva considera que el proceso salud-enfermedad es socialmente determinado, por lo que el concepto "necesidades de salud", se amplía a todas las cuestiones que se refieren al desarrollo de la vida y que deben conducir al disfrute de una existencia digna (De la Torre, 2015).

Por lo tanto, la Salud Colectiva privilegia en sus modelos o pautas de acción, cuatro objetos de intervención: las políticas (formas de distribución del poder); las prácticas (cambios de comportamiento, cultura, producción de conocimientos, acciones institucionales, profesionales y relacionales); las técnicas (organización y regulación de los recursos y procesos productivos); y los instrumentos (medios de producción de la intervención) (Doctorado en Salud Colectiva, 2002).

La perspectiva del sociólogo francés Pierre Bourdieu (1930-2002) constituye una forma de abordar la realidad social reconociendo la objetividad y la subjetividad que se deposita en los cuerpos y en las cosas. Su teoría sostiene que la sociedad se explica mediante las relaciones de intercambio de capital y que existen formas 
generales de funcionamiento que se pueden analizar independientemente de las características particulares de los individuos. Propone el concepto de "campo" como una herramienta metodológica y como un esquema básico de ordenamiento de la realidad social, cultural y simbólica (Vizcarra, 2002, Bourdieu, 2007). Cada uno de ellos prescribe sus valores particulares y posee sus propios principios reguladores. Además de tratarse de un espacio de fuerzas activas, "el campo es también un campo de luchas tendientes a preservar o transformar la configuración de dichas fuerzas (Bourdieu y Wacquant, 2005, p. 155)" (como se cita por Álvarez\&Sáez\&Ulloa, 2018). En un "campo", los agentes y las instituciones luchan constantemente por ganar el "juego". Las instituciones quieren controlar la situación e imponerse, pero siempre encuentran la resistencia de aquellos a quienes quieren dominar, pues los agentes sociales son poseedores de capitales y, dependiendo de su trayectoria y de la posición que ocupan en el "campo", participan activamente en la preservación del "capital" o en su intercambio.

Bourdieu (2007) define el "capital" como un conjunto de bienes acumulados que se producen, distribuyen, consumen e invierten, y que se ganan o pierden en una lucha histórica por su obtención. La fortaleza de cada "campo" radica en su capacidad para producir, difundir y preservar el "capital". Bourdieu (2005) distingue cuatro tipos: económico, cultural, social y simbólico.

Para el sociólogo francés, es fundamental hacer visibles los mecanismos de poder que las estructuras sociales tienen en las prácticas de los "agentes". Además, plantea la importancia de efectuar un análisis que vincule simultáneamente elementos económicos, culturales, objetivos y subjetivos. Los conceptos de "campo" y "habitus", permiten captar dos modos de existencia de lo social: el primero como lo social hecho cosa (lo objetivo) y el segundo como lo social corporeizado (lo subjetivo). Las prácticas sociales que realizan los "agentes", se explican a partir de la relación dialéctica que existe entre ambos modos (Bourdieu, 2007). La historia juega un papel fundamental y parte de dos supuestos: el mundo social se construye a partir de lo ya construido en el pasado; y las formas sociales del pasado son reproducidas, apropiadas, desplazadas y transformadas en las prácticas y las interacciones de la vida cotidiana de los actores (Bourdieu, 2005; 2007).

Con respecto a la Promoción de la Salud Emancipadora (PSE), en este trabajo retomamos los aportes teóricos de Chapela, quien plantea que esta PSE es la que promueve acciones encaminadas a potenciar las capacidades humanas que impulsen procesos emancipadores para cambiar las relaciones con el poder (Chapela, 2010). Además, reconoce al individuo que envejece, como un sujeto material y subjetivo que puede cambiar su realidad externa e interna. Esto implica que una promoción de salud con estas características, tendrá como objetivos que las personas recuperen su autonomía y genere procesos de la capitalización de los agentes sociales, así como la producción de espacios que faciliten y permitan el cambio. Esto es, propicia que los viejos participen, decidan, construyan, sueñen, planteen proyectos y que se abran los espacios para la construcción de futuros. 
Para llevar a cabo lo anterior, también se requiere de la formulación de estrategias de exigibilidad y justiciabilidad de sus derechos, así como el impulso de procesos autonómicos (Cerda, 2010).

\section{Conceptos esenciales del modelo}

Hemos señalado que, con fines de intervención comunitaria, el envejecimiento tiene su inicio en la quinta década de la vida (alrededor de los 45 años edad), "ya que en esa etapa se presentan cambios biológicos, físicos, psicológicos y sociales patentes relativos al envejecimiento en la mayoría de la población" (MartínezMaldonado \& Mendoza-Núñez, 2015, p. 14).

Entendemos a la vejez como una construcción social y cultural, individual y colectiva, que implica formas de percibir, apreciar y actuar que son generadas en espacios socio-históricos determinados por una cultura y que adopta los significados y características generales de éstos. La vejez es un concepto dinámico que se transforma en el espacio y el tiempo, por lo que optamos por hablar de vejeces y no solo de una vejez, es decir, dado que los individuos somos distintos, las experiencias de envejecer también lo son (Martínez-Maldonado et. al., 2018, Martínez-Maldonado et. al., 2009, Vivaldo, 2017).

Sostenemos que el desarrollo humano es un proceso que debe ubicarse dentro de un contexto socio cultural, mediante el cual las personas despliegan sus capacidades y potencialidades físicas, psicológicas y sociales durante el envejecimiento, para constituir de manera activa y responsable un capital social que busque el máximo de salud y bienestar acorde con sus expectativas y propias decisiones (Mendoza-Núñez, 2015).

Respecto del concepto de envejecimiento saludable, lo definimos como el proceso a través del cual una persona ejerce y desarrolla sus capacidades humanas, avanza en la construcción y logro de sus proyectos, reflexiona continuamente sobre su situación, contexto y posibilidades, por lo que reconstruye lo que ha hecho con sus experiencias pasadas (Chapela, 2015). Con base en lo anterior entendemos a la salud como: la capacidad corporeizada que tenemos todos los seres humana de diseñar, decidir y lograr futuros viables y alcanzarlos (Chapela, 2008).

El Desarrollo Comunitario puede ser entendido como el protagonismo de la comunidad en la toma de decisiones que afecta la vida de sus miembros y de su propia búsqueda de alternativas de desarrollo. Para lograr esta participación activa, los habitantes de un territorio requieren conectarse entre sí, analizar sus problemas, establecer prioridades, generar agendas compartidas y diseñar y operar planes y proyectos para el desarrollo de su comunidad. 


\section{Un modelo de intervención}

Con base en los conceptos anteriores, se diseñó el modelo de intervención para el desarrollo comunitario para el envejecimiento saludable. Lo que se pretende es superar las visiones asistencialistas que no reconocen la importancia de los capitales y la participación de las personas envejecidas en la construcción de los proyectos dirigidos a este grupo (ver figura 1).

El modelo está integrado por cuatro etapas que guardan una estrecha relación entre ellas y que constantemente están sometidas a procesos de evaluación. Las cuatro se basan en los aportes de la Salud Colectiva, la Teoría Sociológica de Pierre Bourdieu y la Promoción de la Salud Emancipadora, que tienen como objetivo fomentar el desarrollo de la autonomía, la participación, la agencia y el ejercicio de la ciudadanía a través de la incorporación del autocuidado, la ayuda mutua y la autogestión mediante procesos educativos participativos, con el fin de alcanzar un envejecimiento saludable. A continuación, presentamos cada una de ellas:

- Diagnóstico. Se trabaja en el reconocimiento de la historia, cultura y capitales de la comunidad a través de la realización de un mapeo o inventario de los recursos que han permitido el desarrollo de las capacidades humanas de los miembros de la comunidad. Posteriormente, a partir de las herramientas de la educación participativa, se identifican, junto con los miembros de la comunidad, los problemas y necesidades para resolverlos a partir de procesos formativos e informativos. Este proceso se somete a evaluación a partir de trabajo cuantitativo y cualitativo con base en el entrecruzamiento de fuentes, métodos y técnicas.

- Diseño y planificación de la intervención. A partir del diagnóstico realizado se lleva a cabo, entre los planificadores y la comunidad, el diseño y la planificación de la intervención cuyo objetivo es resolver una necesidad que se traduzca en un beneficio para la comunidad. Se realiza el análisis de la participación, problemas, objetivos y estrategias. Esta etapa también se somete a evaluación utilizando metodologías cuantitativas y cualitativas.

- Intervención. Esta fase se inicia con un diagnóstico del grupo con el que se trabajará para identificar sus capitales, liderazgos e intereses, así como su estado físico, emocional y social. Con lo anterior se describe a la población. Posteriormente se identifican los temas que son de interés particular con el propósito de incluirlos en la planificación previamente realizada. Una vez realizadas estas actividades, se procede a la intervención y se recopilan los datos para la elaboración de los resultados. 
- Evaluación y seguimiento. Este proceso se lleva a cabo aproximadamente seis meses después de la intervención. Se realiza a partir de metodologías cualitativas y cuantitativas y se verifica el impacto de la intervención a partir de los indicadores previstos en la planificación.

El modelo funciona bajo este esquema conceptual. Es importante resaltar que el punto de partida de la propuesta es, fundamentalmente, el fomento de la participación comunitaria en el desarrollo social y en la construcción de sociedades solidarias más prósperas y democráticas. A esto hay que añadir las siguientes líneas, que también guiarán el quehacer del modelo:

- Se concibe como un espacio de diálogo, intercambio y encuentro permanente entre los distintos actores locales, entre comunidades y entre organizaciones comunitarias.

- Se trabaja especialmente la cuestión de identidad: los distintos actores sociales deben sentirse parte de la sociedad local, identificarse con ese entorno y reconocerse en su historia y en su sistema de normas y valores. Es necesario reforzar identidades, así como desarrollar formas de construir identidad alli donde se ha perdido. Dado esto, se dará énfasis a lo relacionado con el rescate histórico y cultural de las comunidades.

- Se hace énfasis en la importancia de que las comunidades, sus grupos y organizaciones trasciendan el nivel de la demanda y accedan al nivel de la propuesta. Cuando se habla de políticas sociales (locales, regionales, sectoriales, etc.), se las asume como una responsabilidad exclusiva del Estado, cuestión que, en la idea de desarrollo local, se busca superar, afirmando que los espacios locales son capaces de generar estrategias y propuestas asociadas a nuevas formas de acción, que pueden contribuir a la diversificación de los agentes potenciales del desarrollo, promoviéndolos, también, al interior de la sociedad civil. Así, las organizaciones sociales de las comunidades, y sus iniciativas, deben ser entendidas, por las propias comunidades, como recursos que deben ser potenciados.

- Se trabaja para que las comunidades y sus organizaciones asuman iniciativas para enfrentar sus problemas, que asuman que todo problema tiene un primer nivel en el que puede ser enfrentado localmente, y que es su responsabilidad hacerlo.

- Se detectan y forman cuadros de liderazgo y de promotores locales y voluntarios.

- Se construyen puentes intergeneracionales, ya que se trata de construir una sociabilidad enriquecida, de valoración de una "escala humana", de valoración de la vecindad y el barrio, de la solidaridad. 
- Se trabaja con base en metodologías participativas, principalmente con el enfoque conocido como centrado en la persona que aprende. Este enfoque ayuda a los que aprenden a asumir un mayor grado de control sobre sus propias vidas y sobre su medio ambiente, mediante el desarrollo de su capacidad para resolver sus problemas y administrar sus recursos

\section{Objetivo general}

Contribuir al mejoramiento de la calidad de vida de las personas envejecidas y en proceso de envejecimiento de las comunidades de algunos municipios del estado de Tlaxcala, México, ampliando sus oportunidades para adquirir conocimientos y habilidades que les permitan un desarrollo humano durante el proceso de envejecimiento, tomando en cuenta sus opiniones y propuestas para implementar estrategias viables enmarcadas en el envejecimiento activo y saludable que permitan mantener y potenciar sus capacidades físicas, psicológicas y sociales en su entorno comunitario para lograr el máximo de salud, bienestar y calidad de vida.

\section{Objetivos específicos}

Que los participantes:

- Adquieran el interés, la constancia y la disciplina que se requieren para iniciar por sí mismos la transformación de sus condiciones de vida.

- Participen activamente para acceder a la capacitación y formación para el trabajo comunitario, así como a diferentes cursos y diplomados con validez oficial.

- Adopten y mantengan estilos de vida saludables en el marco del autocuidado para un envejecimiento saludable.

- Adquieran herramientas, conocimientos y capacidades que les permitan competir en el mercado laboral y ser autosuficientes y productivos.

- Establezcan puentes de comunicación intergeneracional, a través del desarrollo de proyectos que sean de beneficio social amplio.

- Propongan proyectos sociales y productivos que sean factibles de realizar y de los cuales sean ellos los principales actores.

- Influyan positivamente en su entorno social inmediato, beneficiando a sus localidades, sus municipios. 


\section{Características de su operación}

- Propicia el intercambio de experiencias e ideas sobre derechos, deberes y valores, acceso a la salud, educación y cultura, proyectos productivos, organización y participación, entre otros muchos temas, y de fijación de prioridades, producción de materiales locales y estrategias de acción para el desarrollo local y regional.

- Integra actividades físicas, culturales, educativas, de formación, capacitación e investigación.

- Facilita el acceso a información útil a las personas, grupos y organizaciones sociales sobre distintos temas, mediante libros, además de informar sobre cursos y actividades que ofrece y promueve el propio centro y las distintas instituciones del estado.

- Promueve el envejecimiento activo y saludable.

- Brinda asesoría a personas y grupos sobre todos estos temas, y sobre otros que sean propuestos por la propia gente.

- Incluye enseñanza a nivel práctico y comunitario, investigación orientada a la acción, estudios de viabilidad y de diagnóstico de necesidades, y el seguimiento y evaluación de los proyectos colectivos que se instrumenten.

- Forma promotores comunitarios.

- Facilita el intercambio de experiencias e ideas entre las personas, grupos y redes, y entre los distintos actores sociales de las localidades y municipios.

- Promueve el voluntariado dentro de su área de influencia, como una forma más de extender el proceso de organización y participación de las comunidades.

- Incluye a todas las personas, sin distinción de credo, raza, nivel socioeconómico, a grupos vulnerables o minoritarios.

Estas características hacen que el Modelo no sea un espacio estático, sino un espacio en permanente evolución. De ahí la enorme riqueza social que puede aportar a las localidades. 


\section{Resultados}

El diagnóstico, de manera general se lleva a cabo en cuatro sesiones. En ellas se aplican distintas las siguientes actividades participativas: (1) elaboración de mapas y "dos círculos", ambos actividades como muestra de investigación abierta; (2) encuesta participativa, como muestra de una actividad de investigación enfocada (se enfocan a establecer un diagnóstico basado en la percepción de los participantes); (3) "ejercicio de los globos", como actividad de análisis de la situación producto del diagnóstico y (4) "campo de fuerzas", como actividad de planeación participativa, enfocada al trabajo necesario para afrontar los problemas considerados como prioritarios por los participantes. Estas actividades se llevaron a cabo en los siguientes municipios: Santa Chautempan, Atlangatepec, Zitlaltepec, Ixtenco, Santa Cruz Tlaxcala, Tlaxcala capital, Apizaco, Calpulalpan, Contla de Juan Cuamatzi.

Las principales problemáticas detectadas en todas las comunidades fueron las siguientes:

- Alimentación, que consideran es deficiente, producto de no contar, en muchos casos, con ingresos propios o por no contar con apoyos.

- Falta de atención al adulto mayor enfermo.

- Deficientes servicios de salud.

- Transporte (refiriéndose a que son mal tratados por los choferes, que muchas veces no les hacen parada porque dicen que son muy tardados para abordar o descender del vehículo).

- Falta de respeto a los adultos mayores.

- Falta de apoyos gubernamentales a los adultos mayores.

- Imposibilidad de conseguir un empleo.

- Falta de actividades o talleres para los adultos mayores.

- Cuidado de los nietos.

- Exigencias de los hijos.

- Calles y banquetas en mal estado, lo que les dificulta caminar.

- Uso político de los recursos que da el gobierno para los adultos mayores (preferencias partidistas). 
A continuación se presentan los resultados obtenidos en el municipio de Santa Cruz.

Diagnóstico: Las actividades permitieron que los participantes se dieran cuenta de que los problemas afectan por igual a los adultos mayores y al conjunto de la comunidad. Después se buscó que las personas identificaran los recursos con que cuentan para enfrentar y resolver los problemas. Solo identifican una institución, ninguna organización social y no se ven a sí mismos ni a la comunidad como recursos. Los problemas que consideran más importantes son: salud, inseguridad, las malas autoridades, la falta de agua y la contaminación (en este orden). Finalmente, seleccionaron algunos de los problemas prioritarios (salud y agua) y describieron cómo quieren que se modifique y con qué recursos, así como los obstáculos que deben enfrentar y vencer para resolverlo. A continuación, se presenta el análisis del problema salud.

La situación actual del problema salud fue descrita, en términos generales, de la siguiente forma: Falta de servicios médicos, que además son deficientes porque no hay suficiente personal, sobre todo en el turno nocturno; que no hay ambulancia ni medicamentos suficientes. En la discusión para describir esta "situación actual", el grupo fue abordando el problema de otras maneras, hasta llegar a una visión que no es sólo responsabilidad de las instituciones que brindan el servicio, sino que también los incluye a ellos, ya que se dieron cuenta que hay muchas cosas que pueden hacer por sí mismos, como informarse, prestar apoyo voluntario en la clínica y aprender sobre auto cuidado y enseñar a otros.

En el proceso de identificar recursos, se dieron cuenta que nunca se consideran a sí mismos y a su comunidad como un recurso, lo que concluyeron, es un error que les hace darse cuenta de que son parte del problema porque no participan en nada, no exigen sus derechos porque no los conocen y porque tienen miedo a expresarse frente a otros. También se planteó que el problema es no sólo que no participan, sino que además nunca se han organizado y que, por lo tanto, no saben actuar conjuntamente. Esto generó mucha discusión y al final reconocieron que esto es cierto. Acordaron que se harían el propósito de aprovechar que se reúnen cada semana para discutir los problemas que enfrentan y buscar maneras de organizarse para solucionarlos, a la vez que tratarían de interesar a otras personas.

Diseño y planificación de la intervención. Una vez que seleccionaron como tema prioritario "la salud" de manera conjunta, se procedió a diseñar la intervención con tres componentes: (1) Capacitación, (2) Investigación y (3) Servicios.

Intervención. En el siguiente cuadro se presentan las intervenciones que se están desarrollando. 


\begin{tabular}{|c|c|c|c|}
\hline \multicolumn{3}{|c|}{ COMPONENTE } & \\
\hline Capacitación & Investigación & Servicio & $\begin{array}{l}\text { Partici- } \\
\text { pantes }\end{array}$ \\
\hline $\begin{array}{l}\text { Taller } \\
\text { Autoestima en adultos } \\
\text { mayores }\end{array}$ & $\begin{array}{l}\text { Impacto de un programa } \\
\text { de empoderamiento en la } \\
\text { autoestima de personas mayores } \\
\text { de } 45 \text { años de San Miguel Contla, } \\
\text { Tlaxcala }\end{array}$ & $\begin{array}{l}\text { Curso } \\
\text { Habilidades de } \\
\text { autocuidado }\end{array}$ & 20 \\
\hline $\begin{array}{l}\text { Taller } \\
\text { Uso de herramientas } \\
\text { tecnológicas durante la } \\
\text { vejez }\end{array}$ & $\begin{array}{l}\text { Impacto de un programa de } \\
\text { fortalecimiento de la inclusión } \\
\text { de las personas envejecidas en el } \\
\text { uso de herramientas tecnológicas } \\
\text { en la memoria de trabajo de las } \\
\text { personas envejecidas de Santa } \\
\text { Cruz Tlaxcala }\end{array}$ & $\begin{array}{l}\text { Grupo de } \\
\text { reflexión de } \\
\text { cuidadores no } \\
\text { formales }\end{array}$ & 30 \\
\hline $\begin{array}{l}\text { Taller } \\
\text { El arte, la cultura y } \\
\text { la recreación para el } \\
\text { fortalecimiento de la } \\
\text { participación durante la } \\
\text { vejez }\end{array}$ & $\begin{array}{l}\text { Impacto de un programa de } \\
\text { arte, cultura y recreación en la } \\
\text { participación de personas en } \\
\text { proceso de envejecimiento en } \\
\text { Santa Cruz Tlaxcala }\end{array}$ & Feria cultural & 18 \\
\hline $\begin{array}{l}\text { Taller empoderando tu } \\
\text { camino. }\end{array}$ & $\begin{array}{l}\text { Trayectorias individuales de vida. } \\
\text { Estudio cualitativo. }\end{array}$ & $\begin{array}{l}\text { Asesoría de } \\
\text { proyectos } \\
\text { productivos }\end{array}$ & 5 \\
\hline $\begin{array}{l}\text { Taller } \\
\text { Autogestión en la vejez. }\end{array}$ & $\begin{array}{l}\text { Impacto de un programa de } \\
\text { formación de promotores } \\
\text { institucionales para el } \\
\text { envejecimiento saludable. }\end{array}$ & Curso de Tai chi & 10 \\
\hline $\begin{array}{l}\text { Taller } \\
\text { Discriminación y } \\
\text { derechos humanos } \\
\text { durante la vejez. }\end{array}$ & $\begin{array}{l}\text { Efectos de los estereotipos } \\
\text { negativos de la vejez en la salud } \\
\text { de personas en proceso de } \\
\text { envejecimiento en San Miguel } \\
\text { Contla Tlaxcala. }\end{array}$ & $\begin{array}{l}\text { Grupo de ayuda } \\
\text { mutua }\end{array}$ & 20 \\
\hline $\begin{array}{l}\text { Taller } \\
\text { Fortalecimiento de la } \\
\text { autoestima y el desarrollo } \\
\text { de la generatividad. }\end{array}$ & $\begin{array}{l}\text { Relación entre el desarrollo de la } \\
\text { generatividad y la participación } \\
\text { social. }\end{array}$ & $\begin{array}{l}\text { Curso: } \\
\text { Formación de } \\
\text { promotores } \\
\text { gerontológicos }\end{array}$ & 15 \\
\hline $\begin{array}{l}\text { Taller } \\
\text { Tecnologías para la } \\
\text { gestión comunitaria. }\end{array}$ & $\begin{array}{l}\text { Efecto del uso del celular y la } \\
\text { computadora en las relaciones } \\
\text { intergeneracionales. }\end{array}$ & $\begin{array}{l}\text { Curso } \\
\text { Uso del celular }\end{array}$ & 10 \\
\hline
\end{tabular}


Evaluación y seguimiento. Para esta fase cotidianamente se está recuperando información a partir de diferentes instrumentos como fotografías, cuestionarios, autoevaluaciones, demostraciones, videos, relatorías, diarios de campo, documentos que se generan durante las sesiones, entre otros.

\section{Conclusiones}

El diseño del modelo es de reciente manufactura. Se desarrolló al interior de la Licenciatura en Desarrollo Comunitario para el Envejecimiento en Tlaxcala, México. Su aplicación se llevó a cabo por los mismos estudiantes de dicha licenciatura en comunidades del estado, bajo la supervisión de los profesores responsables de las asignaturas relacionadas con investigación e intervención comunitaria. La importancia de articular teorías sólidas, con el modelo de desarrollo comunitario, se refleja en los resultados obtenidos hasta el momento. El modelo muestra ser factible y viable, toda vez que promueve ambientes propicios para la reflexión de las personas que envejecen sobre su papel social y condición ciudadana. Reconocer a la cultura, la historia y la participación de la comunidad como elementos esenciales para la planificación, ofrece la oportunidad de que las personas envejecidas se impliquen en los procesos de capacitación e investigación y participen activamente en el mejoramiento de su salud. El modelo se basa en una implementación sistematizada de cada una de sus diferentes etapas, estando sujeto a una constante evaluación, lo que posibilita implementar adecuaciones inmediatas durante el proceso. 


\section{Referencias}

Álvarez, E., Sáez, F., y Ulloa, J. (2018). Redes de políticas de familia: Una propuesta analítica desde Pierre Bourdieu. Cuhso. Cultura-hombre-sociedad, 28, 1, 128153.

Bourdieu, P. (2000). Cosas dichas. Barcelona: Gedisa. (2005). Intelectuales, política y poder. Buenos Aires: Eudeba. (2007). El sentido práctico. Buenos Aires: Siglo XXI.

Cerda, G. A. (2010). Procesos emancipadores y acción colectiva en salud. En Chapela, M. C. y Cerda, G. A. (Comps.). Promoción de la salud y poder: reformulaciones desde el cuerpo-territorio y la exigibilidad de derechos. México: Universidad Autónoma Metropolitana-Xochimilco.

Chapela, M. C. (2008). ¿Qué promoción de la salud ha fracasado? En Chapela, M.C. y Cárdenas, R. (Coomps.). Aspectos de las políticas y prácticas públicas de salud en México. Seguridad Social, servicios, promoción de la salud. México: Universidad Autónoma Metropolitana.

(2010). Contenidos de poder en la historia de la promoción de la salud. En Chapela, M. C. y Cerda, G. A. Promoción de la salud y poder: reformulaciones desde el cuerpo-territorio y la exigibilidad de derechos. México: Universidad Autónoma Metropolitana-Xochimilco.

(2015). Capacidades para la promoción de la salud y el empoderamiento de las mujeres adultas mayores. En: Martínez Maldonado, M. L., MendozaNúñez, V. M. (2015). Promoción de la salud de la mujer adulta mayor. México: Instituto Nacional de Geriatría.

De la Torre, M. C. (2015). Salud colectiva: fundamentos conceptuales. Revista Salud Areandina, 1, 24-36.

Gutiérrez, J. P., Rivera-Dommarco, J., Shamah-Levy, T., Villalpando Hernández, S., Franco, A., Cuevas-Nasu, L., Romero-Martínez, M., \& Hernández-Ávila, M. (2012). Encuesta Nacional de Salud y Nutrición 2012. Resultados Nacionales. Cuernavaca: Instituto Nacional de Salud Pública.

Gutiérrez, L. \& Lezana, M. (2013). [Coords.]. Propuesta para un plan de acción en envejecimiento y salud. México: Secretaría de Salud/Instituto Nacional de Geriatría.

Instituto Nacional de Estadística y Geografía (2015). Estadísticas a propósito del día internacional de las personas de edad (1 de octubre). Recuperado de www.inegi.org.mx/inegi/contenidos/espanol/prensa/contenidos/ estadisticas/2014/adultos0. pdf? $s=$ inegi\&c=2923\&ep $=170$. 
Martínez-Maldonado, M. L., Chapela, M. M. C., \& Mendoza-Núñez, V. M. (2009). Las prácticas significantes de los viejos y su relación con el programa de envejecimiento activo en una zona de alta marginalidad en México. En Olivo, V.M.G. \& Piña, M.M. (eds.), Envejecimiento y cultura en América Latina y el Caribe (pp.183-197). Santiago: Universidad Central de Chile.

Martínez-Maldonado, M. L., Correa-Muñoz, E. \& Mendoza-Núnez, V. M. (2007). Program of active aging in a rural Mexican community: a qualitative approach. BMC Public Health, 7, 276.

Martínez-Maldonado, M. L., \& Mendoza-Núñez, V. M. (2015). Promoción de la salud de la mujer adulta mayor. México: Instituto Nacional de Geriatría.

Martínez-Maldonado, M. L., Vivaldo-Martínez, M., \& Mendoza-Núnez, V. M. (2016). Comprehensive gerontological development: a positive view on aging. Gerontology \& Geriatric Medicine, 2, 2-23.

Mendoza-Núñez, V.M. (2015). Envejecimiento humano:Un marco conceptual para la intervención comunitaria. En Martínez-Maldonado, M. L., \& MendozaNúñez, V. M. (2015). Promoción de la salud de la mujer adulta mayor, 13-40. México: Instituto Nacional de Geriatría.

Mendoza-Núñez, V.M., Martínez-Maldonado, ML, \& Vivaldo-Martínez, M. (2016). What is the onset age of human aging and old age? International Journal of Gerontology, 10(1), 56.

Mendoza-Núnez, V. M., \& Martínez-Maldonado, M. L. (2013). Modelo de redes sociales y comunitarias para mantener la salud en la vejez. En Montes de Oca, V. (ed.), Envejecimiento en América Latina y el Caribe. Enfoques en investigación y docencia de la Red Latinoamericana de Investigación y Envejecimiento, 453-480. México: Instituto de Investigaciones Sociales, Universidad Nacional Autónoma de México.

Mendoza-Núnez, V. M., Martínez-Maldonado, M. L., \& Correa-Muñoz, E. (2009). Implementation of an Active Aging Model in Mexico for Prevention and Control of Chronic Diseases in the Elderly. BMC Geriatrics, 9, 40.

Mendoza, J. y Jarillo, E. (2011). Determinación y causalidad en salud colectiva. Algunas consideraciones en torno a sus fundamentos epistemológicos. Ciencia \& Saúde Coletiva (16), 847-854.

Universidad Autónoma Metropolitana Xochimilco. (2002). Doctorado en Ciencias en Salud Colectiva. Plan de Estudios. México: UAM-Xochimilco.

Vivaldo, J.P. (2017). Los ancianos en la ciudad de México. Interpretaciones históricas de la vejez (1876-1910). México: Universidad Nacional Autónoma de México (tesis de doctorado). 
Vizcarra, F. (2002). Premisas y conceptos básicos en la sociología de Pierre Bourdieu. Estudios sobre las culturas contemporáneas, 8, 55-68.

World Health Organization. (2002). Active Ageing. A Policy Framework. WHO, Geneva. Recuperado de http://whqlibdoc.who.int/hq/2002/WHO_NMH_ NPH_02.8.pdf.

World Health Organization. (2015). World Report on Ageing and Health. WHO, Geneva. Recuperado de http://apps.who.int/iris/ bitstream/10665/186463/1/9789240694811_eng.

Dirección de correspondencia:

Mạ de la Luz Martínez Maldonado. Psicóloga, UNAM. Maestra en Gerontología Social, UNAM. Doctora en Ciencias en Salud Colectiva, UNAM Académica Universidad Nacional Autónoma de México

Contacto: marilumtz05@gmail.com 